\title{
Formation of Technological Cognitive Reason with Artificial Intelligence in Virtual Space
}

\author{
Evgeniy Bryndin \\ Research Center "Natural Informatic", Scientific Department, Russia, Novosibirsk \\ Email: bryndin15@yandex.ru
}

\begin{abstract}
:
Reason is the basis of our knowledge. It characterizes the mindfulness of thought activity, the ability to think universally, the ability to analyze, abstract, and generalize. Thanks to reason, computers, information technology, artificial intelligence, digital twins, cognitive robots have emerged. Using advances in science and technology, the international scientific and engineering community is trying to create an information technology mind. At the current stage of development of information technologies, cognitive robotics, digital twins and artificial intelligence systems, their synergy allows to begin to form a rational cognitive mind with artificial intelligence in virtual space. A cognitive virtual mind with artificial intelligence can be formed by a cognitive ensemble of intelligent mobile diversified agents with strong artificial intelligence through the regenerative development of professional skills, enhancing them visual, sound, subject, spatial, and temporal sensitivity.
\end{abstract}

Keywords:

cognitive virtual mind; artificial intelligence; cognitive ensemble; intelligent mobile diversified agents

\section{Introduction}

In terms of cybernetics, the artificial mind is the ability to extract and recycle information, the ability to create models of the outside world. This requires a system capable of learning as a necessary stage in the accumulation of reasonable information, and creativity as a purely human acquisition. Then the artificial mind, as an artificial modeling system, can be compared to the natural mind in the quality of information processing.

Scientists have realized that the extraction and processing of information is an objective process. It can be played on computers separately from the person. Engineers have learned to create artificial systems that approach humans in intelligence. It is possible to create an artificial mind more effective than a human mind, whose capabilities are rather limited by the speed of information processing, slow learning, difficulty of fast recollection and sampling of information

The term Digital Twins appeared in the early 2000s, but every year, as technology developed, it received new content. The basic concept is not difficult to understand: monitoring of a physical object is carried out on the basis of a closed cycle of information exchange between it and its virtual model (thus a digital twin).

A digital twin is a virtual prototype of a real object, group of objects, or processes. It is a complex software product that is built from a wide variety of data. The digital twin is not limited to collecting data obtained during the development and manufacture phase of the product - it continues to collect and analyze data during the entire life cycle of the real object, including through numerous IoT sensors. A digital twin is a virtual reproduction of the operational state of a real physical object, process, system, or whole service. It can be a virtual 
twin of a part, product, equipment, process, production sites, workshops, or even factories. It is essentially a set of mathematical models describing the state of an object and all its elements. In general, a digital twin includes: a geometric model of an object; Set of calculated data of parts, nodes and object as a whole (mathematical models describing all physical processes taking place in the object); Information on manufacturing and assembly processes of individual elements; Some data on tests of the object, for example, readings of sensors, from which calculated data can be confirmed; A PLM system that links all of the above objects into a single structure.

The digital twin is used in association with the physical throughout the life cycle: during the testing, rework, operation and disposal phase. The physical object uses sensors that collect real-time data about the state of the object, after which this information is sent to the digital twin. On the basis of the obtained data, the digital model is clarified, which, in turn, gives recommendations for optimization of the operation and maintenance mode of the real object.

The digital twin allows you to simulate in virtual space the change in the state and characteristics of the entire product when the characteristics of any of its elements change. Its main task is to allow real-time management of all factors affecting the cost and quality of the product even before its production begins. Digital twins are created to significantly accelerate the time to market of new products, so you can present all stages of its life cycle in a virtual environment. Another function of the digital twin is to inherit product data when modifications are made to it. In other words, to maximize the use of previous experience in designing, manufacturing, and testing new product modifications. In this case, we take into account the peculiarities of the product operation and on the basis of the data obtained from the digital twin, we can improve the characteristics of the product modification.

The main advantage is the speed of making technical decisions and the cost of obtaining the required characteristics of the product. Having a digital twin, it is possible to reduce by an order of magnitude the number of natural tests, the number of attempts to work out technological processes, all that is connected with the production of a real material part and its tests, the cost of which is much higher than the cost of mathematical modeling. Another advantage is the possibility of collective work on the product of geographically remote collectives and engineering centers. At the same time, the necessary scientific potential and labor resources can be used than with manual design technology.

If we consider the digital twin not of a specific product, but of the whole production, the advantage is the possibility to simulate in a virtual environment all processes, to determine the necessary quantity and optimal location of equipment depending on the volume and range of products produced. At the same time, if a digital twin is developed for the newly created production, it is possible to identify possible risks and shortcomings through simulation of its work, to correct the project. The digital twin of existing production allows you to work out the implementation or change of technological processes without real interference with the work.

Digital twins allow you to model a variety of situations that can occur in production. Thus, the digital twin allows you to select the most adequate process scenarios to avoid failures and force majeure. Digital twins help improve cloud platform efficiency, solve design challenges early, train employees, support innovation, and more [1-2]. 
There are two main approaches to learning intelligent systems: training of artificial neural network, and professional retraining based on associative communicative logic [3-4]. A person develops intelligence based on feeling - knowledge. New feeling - knowledge contributes to the development of skills and accumulation of behavioural and professional experience, increasing the substantive, spatial and temporal sensitivity of the visual system. The feelings of a person with acquired skills learn and accumulate professional experience and competence. Based on skills and experience, a person begins to feel new professional situations. This helps him acquire new professional skills. Reason conceptually defines them in the form of knowledge. A person begins to accumulate new professional experience. Similarly, it is possible to enhance the professional experience and sensitivity of a cognitive robot with communicative associative technological thinking and adaptive behavior by artificial intelligence tools and analytical systems on technology platforms. Cognitive robot, increasing visual, substantive, spatial and temporal sensitivity, is able to acquire new skills and accumulate professional experience and behavior with the necessary technical means [5-8]. Learning new skills can be done in a virtual space and then developed in a specific environment. Thus, experience of cognitive robot behavior in various specific environments is gradually accumulated. Artificial intelligence can accumulate experience in virtual space by modeling professional activities.

Synergy of digital twins, systems of their retraining, cognitive robots with artificial intelligence and ensembles of intelligent agents allow to begin creation of cognitive mind with artificial intelligence in virtual space with technological singularity. The technological singularity of the artificial cognitive mind will be controlled by the person based on the results of self-improvement in the mode of simulation in virtual space.

\section{Review of Literatures}

\section{Implementing the Cognitive Virtual Mind \\ 2.1 Cognitive ensembles of mobile diversified agents}

Process automation, cloud technology, machine learning, big data analytics and the Internet of Things have changed the fundamental pillars of the high-tech sphere, according to Deloitte's consulting firm, "The Future of Working in the World of Technology. The Harvard Business Review Analytic Services study marks the race for technology across all industries. The digital transformation of the high-tech industry and social sphere currently requires rapid reactions, diversification and flexible mobility. Mobile diversified collaboration agents are beginning to demonstrate the ability to work together effectively. Cognitive ensembles of mobile diversified agents use information from technology platforms such as the European Technology Platform Future Manufacturing Technologies, and analytics platforms such as Visiology, which enables the collection, analysis, and visualization of large amounts of data to be quickly and efficiently addressed. Fast, efficient data collection and analysis of large volumes of data, flexible operational mobility of data updates and synergistic open cooperation of mobile diversified agents with information platforms and analytical systems will help accelerate the digital transformation of the high-tech industry and social sphere by teaching new skills. Learning new skills can be done in a virtual space and then developed in a specific environment. The accumulated experience of professional activity in virtual space is used by cognitive ensembles of mobile diversified agents to develop artificial intelligence.

The cognitive ensembles of agents are an actively evolving direction of artificial intelligence that is still in its formative stages. Work in cognitive ensembles requires attracting knowledge and technology from a number of areas that were previously out of sight of artificial intelligence professionals. The synergy of cognitive ensembles, technological 
platforms and analytical systems brings a number of fundamentally new properties and possibilities to the formation of the cognitive virtual mind, and is essentially a qualitatively new, higher level of its development, the level that allows to predict its leading position in the coming decades [1-8]. Artificial intelligence professionals have a leading role here. This direction is a new interdisciplinary science of synergistic artificial intelligence, in which new systemic qualities and technological singularity arise in the process of joint action and mutual adaptation.

\subsection{Technological Platforms}

Technology platforms are a partnership mechanism aimed at rapid development of research and development by creating a basis for interaction between participants and the most significant developments for development, forming answers to strategic technological challenges. Technology platforms are platforms for implementing the relationships of applied research and technology transfer. They bring together stakeholders and provide dialogue, mobilize and direct existing research and development opportunities, identify new scientific and technological opportunities for modernization, and promote a more effective approach to innovation. They are focused on using best practices and expertise in the global space, focusing interdisciplinary research on linking promising industry technologies to the competencies of the future.

Principles of interaction with technological platforms: openness to join and information transparency; increase in awareness; identity of organizational structure; internationality. Participants in technology platforms may include research institutes and the academic community; researchers; Industry and other regulatory structures. Connections to technology platforms can be horizontal, vertically oriented, distributed, research multi-crossdisciplinary and sectoral.

Technologically advanced countries such as the United States, Germany, France, Great Britain, Japan, Israel, China, Australia, Singapore, Canada, Sweden, Belgium are predominantly with currently developing technology platforms. US innovation strategies are developed on the basis of technology platforms. The University of Manchester (UK) has brought together transnational corporations from different industries (Astra-Zeneca, IBM, BP, Syngenta). ETP 's European technology platforms deal with technology issues that could potentially contribute to a number of key challenges essential for the development and adoption of modern technologies, the creation of technologies for the sustainable development of society, social goods and services based on advanced technologies, technological breakthroughs needed to remain at the forefront of high technology sectors and the restructuring of traditional industry. ISTOK-SOYUZ project ("Information Society Technologies to Open Knowledge for Eastern Europe and Central Asia") is supported by the European Commission. The aim of the project is to provide new opportunities for cooperation in the field of ICT (information and communication technologies) between the European Union (EU), Eastern Europe and the countries of Central Asia (EECA), as well as to facilitate the information programs of the European Union in the field of ICT, including partners from the above-mentioned countries, in particular Russia, Ukraine, Belarus, Armenia and Kazakhstan. ARTEMISIA European Technology Platform for Research and Development in Advanced Research and Embedded Systems Technologies. It was established as early as 2007 by Daimler, Nokia, Philips, STMicroelectronics and Thales.

Development of international strategic alliances in the field of development of synergistic artificial intelligence, within the framework of which the formation of cognitive ensembles of mobile diversified agents is stimulated [1-8]. 


\subsection{Development of Mindfulness by Cognitive Ensembles}

Cognitive ensembles of mobile diversified agents have a well developed and replenished information model of the outside world due to the presence of BZ, reasoning and analysis of actions. Agent mobility is the ability to migrate across technology platforms in search of the necessary information to access analytical systems for analysis.

Cognitive ensembles contain a variety of mobile diversified agents distributed within the system, which migrate through it in search of relevant data, knowledge, procedures on technological platforms and analytical systems and co-operate to achieve their goals. The cognitive behavior of the agent is provided by the ability to take actions. The architecture of the cognitive ensemble allows to use self-learning agents, the principles of which are formed in the process of solving practical ones.

The interaction of agents establishes bilateral and multilateral partnership between the ensemble, technology platforms and analytical systems. It is a prerequisite for building virtual communities. Interaction is accompanied by mutual transformations of agents themselves and relations between them. The main characteristics of interaction are orientation, selectivity, intensity and dynamism:

- Crientation-positive or negative; cooperation or competition; cooperation or confrontation; coordination or subordination, etc.;

- Selectivity - interaction takes place between agents that in any way correspond to each other and the task. The agents may be bound in an ordinary relation and independent in another;

- Intensity - interaction between agents is not related to presence or absence, but is characterized by determined force;

- Dynamism - the direction of mutual actions can change over time.

Agent interaction analysis includes the following tasks:

- identification of the situation of interaction of agents;

- allocation of core roles and their distribution among agents;

- determination of the number and types of interacting agents;

- building a formal interaction model;

- defining a set of possible strategies for agents 'behavior;

- formation of multiple communicative actions.

Each agent has a limited set of knowledge needed to achieve his or her own and common goals. Commitments are one of the tools that enable you to organize the singular mutual actions of agents. They allow you to anticipate the behavior of other agents, predict the future and plan your own actions. The following groups of obligations can be distinguished: (a) obligations to other agents; B) the agent's obligations to the group; C) the group 's obligations to the agent; D) obligation of the agent to himself. The formalized presentation of goals, commitments, desires and intentions, as well as all relevant characteristics, forms the basis of the mental model of the online mobile diversified agent, which ensures its reasonable behavior.

Different forms of interaction between agents arise:

- simple cooperation, which involves integrating the experience of individual agents (assignment of tasks, sharing of knowledge, etc.) without special measures to coordinate their actions;

- coordinated cooperation when agents are forced to coordinate (sometimes involving a special coordinating agent) in order to use resources and their own expertise effectively; 
- productive collaboration where agents share resources or solve a common problem by sharing experiences without interfering with each other

A competitive model is used as a reasonable model for coordinating agents 'behaviour. In the process of teamwork of mobile diversified agents, many tasks are solved:

- recognition of the need for cooperation;

- selection of suitable partners;

- possibility of taking into account the interests of partners;

- organization of negotiations on joint actions;

- development of joint action plans;

- synchronization of joint actions;

- task decomposition and division of duties;

- identifying conflicting targets;

- competition for shared resources;

- formation of rules of conduct in the collective;

- training in collective behaviour, etc.

A feature of the collective behavior of mobile diversified agents is that their interaction in the process of solving private tasks (or one common) creates a new quality of solving these problems. To do this, mobile agents can leave the client server and change to the remote server to perform their actions, so that they can return. The use of mobile agents provides:

- reducing the time and cost of data transmission;

- expansion of limited local resources;

- coordination simplification;

- perform asynchronous calculations.

The life cycle model of mobile diversified agents includes the following steps:

- processing of new messages;

- definition of rules of conduct;

- performance of actions;

- updating the mental model according to the specified rules;

- planning of actions based on preferences and utility.

The mental model includes a description of the purposes, preferences, usefulness, obligations and capabilities, and rules of conduct of agents. Based on this model, different types of intelligent mobile diversified agents are selected. In Russia there are systems of agents Autonomy and WebCompass, designed for intelligent communication and processing of information on the Internet.

When using mobile agents, a number of serious problems must be solved, including: the legality of ways to move agents over the network; verification of agents (e.g. protection against problems); respect for private property rights; maintaining confidentiality of information; overpopulation of network of agents; compatibility between the agent code and the firmware of the network machine.

The main efforts to improve the mindfulness of online mobile diversified search agents on the Internet are aimed at developing models of knowledge representation, methods of inference of new knowledge, models of reasoning and methods of agent training to ensure full interaction of mobile smart agent ensembles with technological platforms and analytical systems [1-8]. 


\subsection{Artificial Intelligence Preferences}

Artificial intelligence achieves preference-based goals. To identify preference on a plurality of objects A is to specify a plurality of all those pairs of objects $(a, b)$ for which object $\mathrm{a}$ is preferable than $\mathrm{b}$. When a preference is identified, the following approaches are possible 1 , 2.

1. Unconditional table-based approach.

We will fill in the table according to the principle:

Aij $=1$ if the ith object is better than the $j$ object;

Aij $=0$ if the ith object is worse than the $j$ object.

2. Logical approach.

The approach comprises three stages:

- private criteria for preference selection are identified;

- table of "alternatives-private criteria" is drawn up, which specifies for each alternative the values of quantitative private criteria or the rank of qualitative criteria;

- critical rule is chosen to determine the best alternative.

Since the private criteria under consideration are qualitative, they are given ranking (by preference) rather than quantitative. Rank scores can be considered as scores. On the basis of them, it is necessary to determine the preference. For this purpose, a decisive rule is created. For example, points 1, 2, 3 .

1. Absolute preference. Alternative ai is preferred to alternative aj if, for all particular criteria, ai is preferred or equivalent to aj. Absolute preference has the property of transitivity (if $\mathrm{A}$ is preferred to $B$ and $B$ is preferred to $C$, then $A$ is preferred to $C$ ).

2. Majority rule preference. Alternative ai is better than aj if the number of private criteria by which ai is better aj is greater than the number of criteria by which ai is worse aj.

3. The criterion of the highest sum of points. Instead of quantifying private criteria, it is possible to set their rank values. The rank value is treated as a score, with the lowest score being 1 for the worst value and the highest score for the best value. The preference criterion is then formulated as: alternative ai is better than alternative aj if the sum of the score estimates for ai is greater than for aj

When using the rule preference criteria or the sum of scores, an additional requirement is often imposed on the alternative - the absence of a private criterion with the worst value. Such alternatives are immediately excluded from consideration. With a large number of alternatives and particular criteria, it becomes difficult to directly determine the best alternative by the majority criterion because of the difficulty of calculating the number of best and worst criteria for each alternative. In this case, a preference table should be drawn up to identify the best alternative. According to the rule of majority and absence of the worst value, a preference table for alternatives is drawn up: if alternative $b$ is preferable to $a$, then at the intersection of row $b$ and column $a, 1$, otherwise 0 is set.

\subsection{Useful Choice of Cognitive Virtual Mind}

The concept of "utility" was introduced into economic science by the English philosopher Jeremiah Bentham (1748-1832). Today, all the science of a market economy is essentially based on two theories: utility and cost. The utility category explains the operation of the law of demand. For example, digital human twin with artificial intelligence analyzes unrealized demand for high-tech products on the market. Unrealized demand for high-tech products in the market in practice is related to the use of key indicators of economic efficiency NPV, IRR, PB, PL, ROI and others. According to the main indicators of economic efficiency, 
the digital twin determines the preferences and usefulness of participants in unrealized demand for high-tech products. It identifies new competencies and skills of technological software functional realization of goods or services to quickly meet demand with minimum production costs.

A useful choice of cognitive virtual mind is a functionality that determines preferences on some set of possibilities by the utility criterion. The cognitive virtual mind develops the ability to highlight the properties and functions of entities regardless of the different conditions in which they are observed, relying on useful choices. The better the cognitive virtual mind begins to distinguish similarities with other adjacent entities, the sooner it gains the skill of generalizations. The logical method as a practical acceptance of the use of logical laws and rules in a particular kind of mental activity of the cognitive virtual mind turns them into an algorithm of logical rational thinking. When logical techniques are used, it turns general logic into application logic. For this purpose forms a set of reasonable possibilities: situations that may arise in a virtual application environment. Also forms a set of originationsexecution of rules and operations in the virtual application environment. And forms set of cognitive functions capable of solving the problem of promotion from the starting situation to the target situation. The path of promotion to the target state is built according to the rules and operations of generation in the applied virtual environment by cognitive functions, using methods of analogy, similarity, combination of available solutions and increase of sensitivity of artificial intelligence. In this way of intellectual activity, the cognitive virtual mind establishes reasonable targeted sequences, forming a new knowledge in the mental model by analysis, synthesis, analogy, comparison, induction, derivation and creative ensembles from well-trained artificial neural networks to achieve the desired goal in dialogue with a professional expert.

\section{Discussion}

\subsection{Approach to the Safe Use of the Cognitive Virtual Mind}

The technological singularity of the cognitive mind with artificial intelligence, neural networks, and machine learning make it difficult to explain how a trained system makes a decision. Because of this, the price of error in the fields: medicine, defense, judicial practice, etc. Artificial intelligence specialists adjust artificial neural networks with the help of coefficients during their training with framed data to obtain the necessary result. In order to safely use cognitive mind with artificial intelligence and artificial neural networks, it is necessary to determine the range of change of attributes of input data and to monitor possible results in simulation mode. To apply results safely everywhere, standardization must be introduced. Briefly we will consider approach to standardization on use of cognitive virtual mind in ISO/IEC 22989 - Information Technology - Artificial Intelligence - Artificial Intelligence Concepts and Terminology.

It Is Necessary To Add The Term And Definition Of Cognitive Virtual Mind With Artificial Intelligence To The Standard:

- cognitive virtual mind with artificial intelligence is a cognitive ensemble of intelligent mobile diversified agents with strong artificial intelligence through the recurrent development of professional skills, increasing visual, sound, substantive, spatial and temporal sensitivity.

The following types of classification should be added to the section relating the types of classification to the stages of the life cycle of the system with artificial intelligence:

- diversification,

- mobility. 
The terms and definitions of diversification and mobility should be added to the standard:

- diversification - expansion of artificial intelligence functions and mastering of a new type of functionality in order to increase efficiency, quality and functional diversity of the intellectual system;

- mobility is the ability of an intelligent robot to rapidly functional retraining and develop its intelligence.

The definitions of diversification and mobility are consistent with the definitions of the standard:

- life cycle: development of a system, product, service, project or other human-created entity from design to write-off;

- life cycle model: the structural basis of life cycle processes and actions, which also serves as a common reference for linking and understanding.

Images and numbers are taken as simulation data. The environment is perceived through images and scenes. Scenes consist of a number of images. Scenes are static (paintings) and dynamic. Dynamic scenes are characterized by patterns of behavior of objects and objects. Patterns are either described by formulas or represented by a graph (numerically). Occupational safety depends on the spatial, temporal, substantive, visual and audible sensitivity of intelligent mobile diversified agents. Behavior security is provided within image similarity boundaries in a safe environment. Simulation shows that the development of behavioural skills (mobility) and professional skills (diversification) increases sensitivity of environmental perception, reduces risks, increases safety.

It is necessary to adjust the definition of artificial intelligence standard:

Artificial intelligence is the ability of a system to acquire, process, apply, and diversify knowledge based on prior experience with specific problems related to data attribute processing and intelligent system mobility.

Attributes of data. Objects, objects, materials, things, processes, phenomena and other aspects of the physical world have different properties and characteristics. Properties are represented by qualitative attributes. Characteristics are represented by meaningful attributes. A qualitative attribute can be visual or audible. A meaningful attribute can be represented by a number, a language sense value, a visual or audible way, a mathematical or behavioral action, or an algorithm. Meaningful qualitative attributes are big smart data of artificial intelligence.

Big Smart Data (Big SD) is a collection of qualitative and quantitative attributes related in time, space, and subject area. Smart data attributes (numbers and images) form a world view. Big SD attributes of the areas of economics, industrial industries, technology, and professions help build and train artificial neural multilayer artificial intelligence networks to manage, make decisions, and make recommendations to professionals and executives. Applied research modeling helps accumulate Big SD scientific attributes in real time and simultaneously use them to deeply train multi-layer artificial neural control networks to simulate applied research, make decisions, and make recommendations to researchers. By modeling, the optimal (equilibrium safe) state of artificial neural networks of intelligent agents and the limits of attribute values relative to this state are determined. Numeric limits are defined for numeric attribute values. For visual and sound images, the limits of similarity to the optimal image are revealed. The development and use of equilibrium safe systems can be applied as a criterion of companies developing a cognitive virtual mind with artificial intelligence. 


\subsection{The Potential of Cognitive Virtual Mind}

The cognitive virtual mind has the potential of a researcher equalizing the world 's income levels and improving the quality of life of populations worldwide. In the future, it will lead to long-term efficiency and productivity gains. Lower transport and communications costs, improve logistics and global supply chains, lower the cost of trade, stabilize markets and stimulate economic growth. Cognitive virtual mind involves using digital technology to transform production and social processes to become more efficient and efficient. He will use and manage technology to improve the quality of life. It will lead to an increase in safe workers revenge. In an ideal world, the interaction of the cognitive virtual mind with people would ensure mutual understanding and cohesion. A cognitive virtual mind is the key to a better tomorrow.

A cognitive virtual mind will finally change not just what we do, but who we are. It will affect our identity and all related issues: our sense of privacy, our concepts of ownership, our consumption patterns, the time we devote to work and recreation, and how we develop our careers, cultivate our skills, meet people and nurture relationships. It will change our lives and lead to quality self-education, it can lead to an increase in human intellectual potential.

One of the problems associated with cognitive virtual mind is privacy. Tracking and sharing information about us is an essential part of the new connection. Disputes over fundamental issues such as the impact on our internal lives of losing control of our data could intensify. Similarly, a cognitive virtual mind with artificial intelligence that overrides what consciousness means extends the current thresholds of his life expectancy, cognition and opportunity. It will require us to reconsider our moral and ethical boundaries.

A mind with artificial intelligence in virtual space is not an exogenous force over which humans have no control. Developers are responsible for its evolution, in the decisions it will make daily. We must seize the opportunity and achievements we have to shape the mind with artificial intelligence in virtual space and guide it towards a future that reflects our shared goals and values.

To do so, however, we must develop a comprehensive and world-wide view of how the artificial virtual mind must influence our lives and change our economic, social, cultural and human environment. There has never been a time of greater intellectual capability and greater potential danger. Today's leaders are beginning to think strategically about artificial intellectual capabilities that help shape our future.

We need to shape a future that works for all of us, putting people first and empowering them. In a humane form, a cognitive virtual mind can indeed have the potential to robotic humanity's life. And as a complement to the best parts of human nature - creativity, governance - it can elevate humanity into a new collective consciousness based on shared goals. We can make it happen.

\section{Conclusion}

The international scientific engineering community, within the framework of MEGAPROJEKT, can start technical implementation of cognitive virtual mind with artificial intelligence. In the future, in the labor market, sensitive safe robots with cognitive artificial mind will perform high-tech professional work in various areas of industry and social services. A person will teach robots with a cognitive mind to build up intellectuals with recursive selfimprovement to a technological singularity. Self-improving cognitive robots and artificial 
neural networks that process numerical data, as well as visual and sound images, will be able to search for patterns in large amounts of data, recognize objects and people, read texts, make decisions, control movements and others. A cognitive sensitive robot with an artificial mind will be able to train, speculate, apply new knowledge and experience of behavior, and respond wisely and safely to the external environment. A cognitive virtual mind with artificial intelligence is able to develop on the basis of cognitive functions of preference and utility on many reasonable capabilities of technological platforms without predetermined algorithms. Artificial mind with technological singularity will be monitored by self-improvement results in simulation mode in virtual space. He can acquire the status of innovation researcher (EvgeniyKamtini 2020) and become a good assistant to mankind in the safe development of life spaces (Evgeniy, 2020).

\section{References}

Evgeniy Bryndin. Human Digital Doubles with Technological Cognitive Thinking and Adaptive Behaviour. Software Engineering, Volume 7, Issue 1, 2019. P. 1-9.

Evgeniy Bryndin. Collaboration Robots with Artificial Intelligence (AI) as Digital Doubles of Person for Communication in Public Life and Space. Budapest International Research in Exact Sciences (BirEx-Journal), Volume 1, No. 4, 2019. Pages: 1-11.

Evgeniy Bryndin. System retraining to professional competences of cognitive robots on basis of communicative associative logic of technological thinking. International Robotics Automation Journal. 2019; 5(3.):112-119.

Evgeniy Bryndin. Practical Development of Creative Life-Saving Strong Artificial Intelligence. Communications, Vol.7, No.1, 2019. Pages: 31-39.

Evgeniy Bryndin. Development of sensitivity and active behavior of cognitive robot by means artificial intelligence. International Journal of Robotics Research and Development. VOL - 10, ISSUE - 1; 2020. Pages: 1-11.

Evgeniy Bryndin. Robots with Artificial Intelligence and Spectroscopic Sight in Hi-Tech Labor Market. International Journal of Systems Science and Applied Mathematic, V. 4, № 3, 2019. Pages: 31-37.

Evgeniy Bryndin. Development of Artificial Intelligence by Ensembles of Virtual Agents on Technological Platforms. COJ Technical \& Scientific Research.2(4). 2020. Pages: 1-8.

Evgeniy Bryndin. Increased Sensitivity and Safety of Cognitive Robot by Development of Professional and Behavioral Skills. Engineering Mathematics Letters. 2020. In the press.

Evgeniy Bryndin. Technological, Economic and Social Aspects of Management by Development of the Digital Industry 4.0. International Journal of Managerial Studies and Research (IJMSR), vol 6, no. 4, 2018, pp. 19-30

Evgeniy Bryndin. Directions of Development of Industry 4.0, Digital Technology and Social Economy. American Journal of Information Science and Technology. V 2, Issue 1.2018. P. 9-17.

Evgeniy Bryndin. Human Digital Doubles with Technological Cognitive Thinking and Adaptive Behaviour. Software Engineering, Volume 7, Issue 1, 2019. P. 1-9.

Evgeniy Bryndin. Creative innovative transformational ecosystem of formation of humane technological society. International Robotics Automation Journal. 2019;5(3):91-94.

Artificial Intelligence \& Robotics: Industry Report \& Investment Case. 2019. https://indexes.nasdaqomx.com/docs/NQROBO\%20Research.pdf

Evgeniy Bryndin. Robots for Communication in Public in High-Tech Industry Life and Space. Frontiers Journal of Current Engineering Research. Volume 1, Issue 1, 2019. P. 1-10.

Evgeniy Bryndin. Transformation Modeling of Nanostructures by Cognitive Systems according to Big Smart Data. Chepter 3, Pages: 21-28. / Book "New Materials: 
Preparation, Properties and Applications in the Aspect of Nanotechnology". USA: Nova Science Publisher. 2020. 250 p.

Wei Dan, Ren Xian, Ma Qi Hua. Research on the Construction of Courses of Automotive Electronics and Electrical Appliances Based on the Joint Training Mode of Vocational Schools and Applied Undergraduate Courses. Britain International of Humanities and Social Sciences, Vol 2 No 1 (2020). URL: http://doi.org/10.33258/biohs.v2i1.136

Sudirman Sudirman, Rosramadhana Rosramadhana, Ayu Febryani. Gender Education in Disruption Era (Study Of Virtual Ethnography). Budapest International Research and Critics Institute (BIRCI-Journal): Humanities and Social Sciences, Vol 3, No 1 (2020)

Kamtini Kamtini, Damaiwaty Ray, Dorlince Simatupang, Salsabila Hasiana Tanjung, Husni Wardi Tanjung. Project Based Self-Regulated Learning as a Parenting Model to Increase the Attitude of Responsibility of Children Age 5-6 Years. Budapest International Research and Critics in Linguistics and Education (BirLE) Journal, Volume 3, No 1. 2020. DOI: https://doi.org/10.33258/birle.v3i1.753

Evgeniy Bryndin. Cyclical Ecological Economy with Reinvesting of Its Savings. Integrative Journal of Conference Proceedings, 2(2). 2020. P. 1-5.

Evgeniy Bryndin. Financial Turnover of Cyclical Economy by Reinvesting in Ecological Production of Its Savings. J. Resources and Environmental Economics. Volume 2, №. 1. Singapore: Syncsci Publishing. 2020. Pages: 96-101. 